\title{
The influence of the physical properties of the liquid on the formation of thermo-capillary structures
}

\author{
Evgeny Chinnov, Andrey Semenov \\ Kutateladze Institute of Thermophysics SB RAS, 630090, 1 Lavrentyev Ave., Novosibirsk, Russia \\ Novosibirsk State University, 630090, 2 Pirogova Str., Novosibirsk, Russia
}

\begin{abstract}
An experimental study of the formation of thermocapillary structures at low Reynolds numbers and temperature gradients at the surface of the film in a wide range of the viscosity of the liquid was carried out. The data on the distance between the rivulets are generalized. It is shown that the transverse dimension of structures is determined by the capillary constant and does not depend on either the viscosity of the liquid or the flow rate.
\end{abstract}

\section{Introduction}

The flow of a thin layer of liquid (film) over a solid surface belongs to the class of flows with a free surface. Various instability mechanisms play an important role in thees flows, leading to the development and interaction of waves of different scales. Film flows are used in various technological processes and apparatuses. Studies of heat transfer from flat heaters to a flowing liquid film began in the early 90s. In nonisothermal liquid films, there are two rivulet formation regimes [1]. In regime A, high temperature gradients (up to 10-15 $\mathrm{K} / \mathrm{mm}$ ) were observed on the surface of the film in the upper part of the heater. On the surface of the heater, a boundary condition close to $\mathrm{T}=$ const was realized. When the threshold heat flux density was reached, significant deformations appeared on the surface of the film in the upper part of the heater. The stream was divided into vertical rivulets with a certain wavelength $\lambda$. At low Reynolds numbers, high thermocapillary stresses directed against the flow led to a thickening of the film in the form of a horizontal roller [2]. Experimentally, structures of type A were studied for $\operatorname{Re}<25$.

In regime $\mathrm{B}$, the rivulet flow was formed gradually with increasing heat flow and distance from the upper edge of the heater [1]. The boundary condition $\mathrm{q}=$ const was realized on the surface of the heater. The inhomogeneities of the thickness of the film across the flow led to inhomogeneities in the temperature at the surface. The transverse temperature gradient (up to $1 \mathrm{~K} / \mathrm{mm}$ ) resulted in an increase in the deformation of the film surface. An experimental study of the evolution of hydrodynamic waves into thermocapillary-wave structures while heating of a vertically falling film was carried out in [3] for Re $>20$. The fact of the appearance of temperature inhomogeneities at the front of a three-dimensional hydrodynamic wave is shown. Because of the action of thermocapillary forces, these inhomogeneities led to deformation of the film and the formation of rivulets. 
To the present moment, the formation of structures of type B has been studied mainly on two liquids: water and FC-72, with significantly different surface tension coefficients. It is shown that the surface tension is significantly affected by the transverse size of the structures (the distance between the centers of the rivulets).

One of the latest theoretical papers [4] confirms the existence of two types of structures on the surface of a nonisothermal liquid film. It is shown that in the thermocapillary regime the wavy flow decays, and in the thermocapillary-wave regime, the waves propagate along the rivulets. However, the calculations are limited to the region $\operatorname{Re}<5-8$ and do not describe the hydrodynamic instability regime of the liquid wave film. In [5], the thermocapillary rupture of a liquid film flowing under the influence of gravity along a vertical plate with a heater $150 \times 150 \mathrm{~mm}$ is studied in a wide range of fluid properties. It has been established that the viscosity of a liquid has a significant effect on the threshold heat flux at which a film breaks. The effect of the viscosity of a liquid on the characteristics of thermocapillary structures remains undeveloped, especially in the context of small values of the Reynolds number of the film.

The task of this work is an experimental study of the formation of thermocapillary structures with temperature gradients on the surface of the film of less than $1 \mathrm{~K} / \mathrm{mm}$ for small Reynolds numbers over a wide range of viscosity changes.

\section{Experimental setup}

The stand was a closed circulation loop, including a reservoir with a pump, a working section, a filter, rotameters, pipelines and shut-off valves. The working area consisted of a carrier plate, on which a film former, a heat stabilizer and a heater were installed. Using a pump the working fluid was supplied to the film former, which included a storage chamber, a dispenser and a nozzle with a calibrated flat slot. The liquid flowed down the plate and under the influence of gravity, returned to the reservoir with the electric pump. The heating element was an electric heater $150 \mathrm{~mm}$ wide and $150 \mathrm{~mm}$ long. On the surface of the heater, a condition close to $\mathrm{q}=$ const was realized. The following working fluids were used in the experiment: water, $60 \%$ glycerol in water, ethylene glycol. The properties of the liquids are presented in Table 1, where Ka is the Kapitza number $\left(K a=\frac{\sigma^{3}}{g v^{4} \rho^{3}}\right)$, Ma is the Marangoni number $\left(M a=\frac{\gamma \Delta T}{\rho v^{4 / 3} g^{1 / 3}}\right)$, where $\gamma$ is the derivative of the surface tension coefficient on the temperature, the index $a v$ shows that the temperature difference is between the average temperature on the film surface and the initial temperature of the film, the index $l$ shows that the temperature difference is calculated from the maximum temperature drop across the film flow.

Table 1. Working liquids and their properties.

\begin{tabular}{|l|l|l|l|l|l|l|l|l|}
\hline Working liquid & $\mathrm{T}_{0},{ }^{0} \mathrm{C}$ & $\begin{array}{l}\rho, \\
\mathrm{kg} / \mathrm{m}^{3}\end{array}$ & $\begin{array}{l}\nu^{*} 10^{6}, \\
\mathrm{~m}^{2} / \mathrm{s}\end{array}$ & $\begin{array}{l}\sigma^{*} 10^{3}, \\
\mathrm{~N} / \mathrm{m}\end{array}$ & $\begin{array}{l}d \sigma / d T^{*} 10^{3}, \\
\mathrm{~N} /(\mathrm{m} * \mathrm{~K})\end{array}$ & $\mathrm{Ka}$ & $\mathrm{Ma}_{a v}$ & $\mathrm{Ma}_{l}$ \\
\hline Water & 24 & 997 & 0.912 & 72.16 & 0.191 & $5.6^{*} 10^{19}$ & 59 & 11 \\
\hline $60 \%$ glycerol & 24 & 1151 & 7.665 & 67.8 & 0.131 & $6^{*} 10^{15}$ & 3 & 2.4 \\
\hline ethylene glycol & 24 & 1112 & 15.17 & 45.74 & 0.09 & $1.3^{*} 10^{14}$ & - & - \\
\hline
\end{tabular}




\section{Results and conclusions}

Fig. 1 shows the temperature distribution on the film surface of a $60 \%$ solution of glycerin in water (Reynolds number is 1 , the heat flux density is $1.1 \mathrm{~W} / \mathrm{cm}^{2}$ ), where $\mathrm{X}$ is the coordinate counted from the upper edge of the heater, $\mathrm{Z}$ is the coordinate counted from the left edge of the heater. It can be seen that the temperature distribution near the upper edge of the heater is almost uniform. In the conditions of a uniform flow, thermocapillary structures are formed on the surface of the film in the form of rivulets with a thin film between them. Rivulets appear on the surface of the film gradually with increasing mean free path along the heater. The beginning of the formation of the rivulets is approximately $20 \mathrm{~mm}$ from the upper edge of the heater. The longitudinal and transverse temperature gradients on the surface do not exceed $0.5 \mathrm{~K} / \mathrm{mm}$, the temperature difference in the rivulet and the interrivulet region at $\mathrm{X}=130 \mathrm{~mm}$ is of the order of $4^{\circ} \mathrm{C}$, and the distance between the rivulets is $14 \mathrm{~mm}$. In these experiments, the structures were also fixed for different Reynolds numbers from 0.1 to 10 .

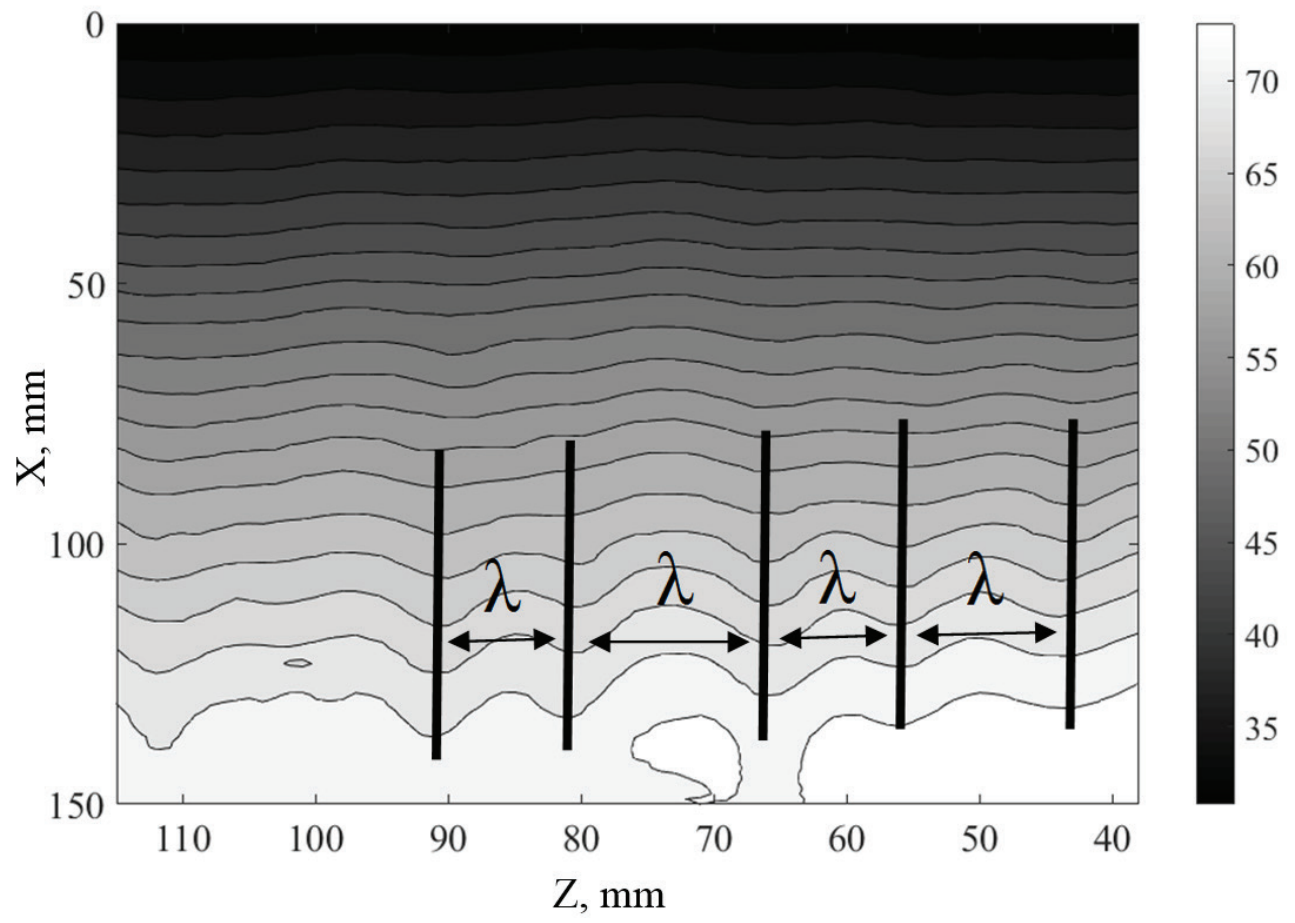

Fig.1 The temperature distribution on the film surface of a $60 \%$ solution of glycerin in water $(\operatorname{Re}=1$, $\mathrm{q}=1.1 \mathrm{~W} / \mathrm{cm}^{2}$ )

For the analysis of data, various dimensionless criteria and parameters were used. Distances were measured for liquids with different densities, surface tension coefficients, and over a wide range of viscosity and fluid flow rates. The distance between the rivulets is dimensioned by dividing by the liquid capillary constant $\left(l_{\sigma}=(\sigma / \rho g)^{0.5}\right.$, where $\sigma$ is the surface tension coefficients, $g$ is the acceleration due to gravity). All data on the dimensions of structures of type B fall in the range from 4 to 6 and does not depend on either the viscosity of the liquid or the Reynolds number. The use of other parameters led to worse results. For example, using the parameter $1_{\delta}=\left(\sigma \mu^{2} /\left(\rho^{3} \mathrm{~g}^{2}\right)\right)^{1 / 5}$, which was used in [7], increased the spread of data by an order of magnitude. 
The theoretical dependence obtained in [6] indicates an increase in the wavelength of the instability (distance between the rivulets) with a decrease in Ma, but the tendency of its increase with increasing Re remains unchanged, which contradicts the experimental data. For Re $>5-8$, calculations based on the theoretical model [6] do not lead to the formation of rivulet flows, while the experimental data obtained indicate a transition from a threedimensional wave flow of an isothermal film to a thermocapillary-wave regime B under a wide variation of the parameters. As follows from Table 1, the range of variation of the Marangoni number was almost 2 orders of magnitude.

It can be concluded that structures of type B are realized at relatively low Marangoni numbers under conditions of small values of the temperature gradients at the surface of the film over a wide range of the Reynolds number of the film and the physical properties of the liquid (Kapitsa number). The transverse size of the structures (the distance between the rivulets) is determined by the capillary constant and does not depend on either the viscosity of the liquid or the flow rate (Marangoni, Kapitsa and Reynolds numbers of the film).

The work was supported by the RF Ministry of Education and Science (Agreement No. 14.604.21.0053, project ID RFMEFI60414X0053).

\section{References}

1. Chinnov E.A., Kabov O.A. Journal of Applied Mechanics and Technical Physics 44 (2003)

2. Kabov O.A. Heat Transfer Research 27 (1996)

3. Kalliadasis S., Kiyashko A., Demekhin E.A. J. Fluid Mechanics 475 (2003)

4. Chinnov E.A., Kharlamov S.M., Saprikina A.V., Zhukovskaya O.V. Thermophysics and Aeromechanics 14 (2007)

5. Chinnov E.A. Technical Physics Letters 34 (2008)

6. Scheid B., Kalliadasis S., Ruyer-Quil C., Colinet P. Physical Review E 78 (2008)

7. Zaitsev D.V., Semenov A.A., Kabov O.A. Thermophysics and Aeromechanics 23 (2016) 